\title{
A spectrofluorometric analysis to evaluate transcutaneous biodistribution of fluorescent nanoparticulate gel formulations
}

\author{
Enrica Cappellozza, ${ }^{1}$ Federico Boschi, ${ }^{2}$ Maddalena Sguizzato,${ }^{3}$ Elisabetta Esposito, ${ }^{3}$ Rita Cortesi, ${ }^{3}$ \\ Manuela Malatesta, ${ }^{1}$ Laura Calderan ${ }^{1}$ \\ ${ }^{1}$ Department of Neurosciences, Biomedicine and Movement Sciences, Section of Anatomy and Histology, School of \\ Medicine and Surgery, University of Verona \\ ${ }^{2}$ Department of Computer Science, University of Verona \\ ${ }^{3}$ Department of Chemical and Pharmaceutical Sciences, University of Ferrara, Italy
}

\begin{abstract}
The investigation of the absorption of drug delivery systems, designed for the transport of therapeutic molecules inside the body, could be relatively simplified by the fluorophore association and tracking by means of bio-imaging techniques (i.e., optical in vivo imaging or confocal and multiphoton microscopy). However, when a fluorescence signal comes out from the skin, its specific detection can be problematic. Skin high autofluorescence can hinder the observation of administered exogenous fluorophores conjugated to drug delivery systems, making it more challenging to detect their biodistribution. In the present study, we have developed a method based on the spectrofluorometric analysis of skin samples to discriminate the fluorescent signal coming from administered fluorescent molecules from the background. Moreover, we gave a semi-quantitative evaluation of the signal intensity. Thus, we distinguished two gel formulations loading the fluorophore rhodamine $\mathrm{B}$ (called GEL RHO and GEL SLN-RHO). The two formulations of gels, one of which containing solid lipid nanoparticles (GEL RHO-SLN), were administered on skin explants incubated in a bioreactor, and the penetration was evaluated at different time points ( 2 and 6 hours). Cryostatic sections of skin samples were observed with confocal laser scanning microscopy, and a spectrofluorometric analysis was performed. Significantly higher signal intensity in the samples administered with SLN-RHO GEL, with a preferential accumulation in the hair bulbs, was found. Reaching also the deeper layers of the hair shaft after 6 hours, the solid lipid nanoparticles thickened with polymer represent a suitable drug delivery system for transcutaneous administration.
\end{abstract}

Key words: Organ culture; bioreactor; transcutaneous biodistribution; spectrofluorimetric analysis.

Correspondence: Federico Boschi, Department of Computer Science, University of Verona, Strada Le Grazie 15, 37134 Verona, Italy. Tel. +39.045.8027272. E-mail federico.boschi@univr.it

Contributions: LC, EC, RC, EE, FB, conceptualization; MS, EE, methodology; EC, FB, data analysis; EC, LC, FB, investigation; EC, FB, LC, MM, original draft preparation; LC, FB, MM, supervision; LC, project administration, funding acquisition. All authors have read and agreed to the published version of the manuscript.

Funding: This research was funded by the University of Verona (Joint Projects 2017).

Conflict of interest: The authors declare no competing interests, and all authors confirm accuracy.

Availability of data and materials: The datasets used and/or analysed during the current study are available from the corresponding author on reasonable request.

Ethical approval: All animal-related procedures were applied according to the project approved by the Italian Ministry of Health (code 56DC9. N.703), under the regulations of the Italian Ministry of Health (DL March 4, 2014, no. 26, directive implementation 2010/63/ EU) and the European Communities Council (Directive 63/2010/EU of the European Parliament and of the Council) directives. 


\section{Introduction}

Transcutaneous drug delivery is an interesting and widely studied administration procedure for both local and systemic therapeutic effects, which avoids the biotransformation and metabolism through the gastrointestinal tract and liver in the case of oral administration. ${ }^{1}$ However, the skin mainly protects the body from external stress and biological or chemical insults, thus intrinsically limiting the transit of drugs to the underlying vascularized connective tissue. To improve drug absorption, different strategies may be followed. For example, appropriate penetration enhancers may increase the hydration of the stratum corneum, thus increasing the skin barrier fluidity ${ }^{2}$ and/or the number of molecules able to interact with the hydrolipidic film of the skin. Among the most used delivery systems are gels that, by forming an occlusive membrane, increase the hydration state of the skin. Gels are often associated with nanovectors., In fact, over the past 20 years, biomedical research has focused on studying nanotechnologies applied to medicine, leading to the development of nanoparticles (NPs) as drug delivery systems for diagnosis and/or treatment of different pathologies. ${ }^{5,6}$ These NPs have amphiphilic chemicalphysical characteristics allowing them to carry both lipophilic and hydrophilic molecules and facilitate a controlled release of the drug. Notably, a novel approach developed to obtain NPs with a suitable consistency for topical application is represented by the direct addition of polymers. As previously published, ${ }^{7}$ a nanoparticulate gel has been produced by adding poloxamer 407 to nanoparticle dispersions, giving rise to a delivery system with optimal thermogelling properties.

A relatively simple way to evaluate the administration of a compound is to label or associate it with fluorophore and track the absorption by bio-imaging techniques ${ }^{8,9}$ using, for example, confocal and multiphoton microscopy. ${ }^{10,11}$ However, the skin is characterized by a high autofluorescence that can hinder the observation of the exogenous fluorophore and the assessment of their accumulation degree. Autofluorescence thus represents a background signal and is commonly defined as unwanted fluorescence. Autofluorescence emission is generated from endogenous fluorophores, ${ }^{11-13}$ such as collagen, elastin, beta-carotene, oils, pigments, and proteins and also, with regard to the skin, keratin, and vitamin D2 and D3, while melanin can act as an inner filter. All of them can cover and affect the signal of fluorescent NPs, ${ }^{11,14,15}$ in particular in layers ${ }^{16}$ and eccrine sweat glands ${ }^{17}$ of the skin where autofluorescence is particularly higher as compared with other organs of the body.

In the present investigation, we explored the potentialities of a spectrofluorometric analysis of confocal images in discriminating from the background and evaluating the fluorescent signal coming from fluorescent molecules after transcutaneous administration on explanted rat skin.

Skin explants were maintained and treated in a bioreactor able to mimic physiological conditions thanks to the continuous culture medium flow, also ensuring controlled conditions of sterility, temperature and humidity during the whole experiment. ${ }^{18}$

Two different gel formulations containing rhodamine, as a fluorophore, were administered on skin samples, and two time points ( 2 and $6 \mathrm{~h}$ ) were taken into consideration to evaluate the different penetration efficacy in time of the two different gel formulations. Both tested gels were made of poloxamer 407, a copolymer constituted of polyoxyethylene- polyoxypropylene units characterized by thermogelling properties. Poloxamer 407 in water generates micellar structures undergoing a sol-gel transition due to concentration and temperature. Remarkably, at low temperatures $\left(4^{\circ} \mathrm{C}\right)$, the poloxamer 407 solution is low viscous, while above its transition temperature, it assumes a semisolid consistency allowing to control drug diffusion through the skin barrier. ${ }^{7}$ In the first formulation, rhodamine was suspended as a free molecule in a gel matrix. In contrast, in the second, it was encapsulated in solid lipid nanoparticles (SLNs), which were then viscosized by the addition of poloxamer 407 , to evaluate differences in the transcutaneous administration with both formulations.

By thespectrofluorometric method presented in this study, we could discriminate the rhodamine fluorescence signal from the skin autofluorescence while obtaining quantitative estimates of the fluorescence intensity in the different skin layers and cutaneous annex. In particular, the hair bulbs were considered preferential absorption pathways in the transcutaneous penetration of substances. ${ }^{19,20}$

\section{Materials and Methods}

\section{Gel formulation}

We investigated two different formulations loading rhodamine B (Sigma-Aldrich, Milan, Italy) and based on poloxamer 407 (BASF, Ludwigshafen, Germany) as polymer gel matrix. For the first formulation, called GEL RHO, poloxamer 407 gel was prepared by the "cold method", ${ }^{21}$ adding a weighed amount $(15 \%$ $\mathrm{w} / \mathrm{w})$ of polymer to cold water $\left(5-10^{\circ} \mathrm{C}\right)$ under magnetic stirring. Then, rhodamine B $(0.2 \mathrm{mg} / \mathrm{mL})$ was added to the produced gel under vortex motion at $30 \mathrm{~Hz}$. The container was sealed and left in a refrigerator at $5^{\circ} \mathrm{C}$. For the second formulation, SLNs containing rhodamine B were firstly prepared and then thickened with poloxamer 407 . Briefly, the aqueous phase $(4.75 \mathrm{~mL})$ composed of poloxamer $188(\mathrm{BASF})(2.5 \% \mathrm{w} / \mathrm{w})$ heated at $80^{\circ} \mathrm{C}$ has been added to the molten lipid phase $(250 \mathrm{mg})$ constituted of tristearin (SigmaAldrich) and rhodamine B $(0.2 \mathrm{mg} / \mathrm{mL})$. The two phases were mixed with ultra turrax (IKA T25 digital) at $15,000 \mathrm{rpm}$ at $80^{\circ} \mathrm{C}$ for $1 \mathrm{~min}$ and then homogenised by ultrasound at $6.75 \mathrm{kHz}$ for 15 min and stored at $25^{\circ} \mathrm{C}$. Finally, poloxamer $407(15 \% \mathrm{w} / \mathrm{w})$ was directly added to SLNs and magnetically stirred for $3 \mathrm{~h}$, obtaining a nanoparticulate gel called GEL SLN-RHO. The container was kept at $5^{\circ} \mathrm{C}$ to complete dispersion of poloxamer. ${ }^{7}$ We found that GEL-RHO and GEL-SLN-RHO maintained their macroscopical aspect for 6 months from production, stored at 25 and $4^{\circ} \mathrm{C}$. The formulation aspect was homogeneous and free from separation phenomena in all cases.

\section{Sample preparation}

Skin samples were explanted from the abdominal region of one healthy 3-month-old male Wistar rat, previously sacrificed with an overdose of $\mathrm{CO}_{2}$ and shaved on the ventral region. All animalrelated procedures were applied according to the project approved by the Italian Ministry of Health (code 56DC9. N.703), under the regulations of the Italian Ministry of Health (DL March 4, 2014, no. 26, directive implementation 2010/63/EU) and the European Communities Council (Directive 63/2010/EU of the European Parliament and of the Council) directives.

Freshly excised skin samples were rapidly washed in sterile physiological solution $(0.9 \% \mathrm{NaCl} \mathrm{w} / \mathrm{v})$ and pre-warmed culture medium at $37^{\circ} \mathrm{C}$. Culture medium was composed of DMEM (Dulbecco's Modified Eagle's Medium) supplemented with 4.5 $\mathrm{g} / \mathrm{L}$ of D-Glucose, $10.0 \%$ of FBS (Fetal Bovine Serum), $2.0 \%$ of penicillin-streptomycin, $200 \mathrm{mM} \mathrm{L}$-glutamine and $0.3 \mu \mathrm{g} / \mathrm{mL}$ of Amphotericin B (Gibco, Waltham, MA, USA).

Samples with a diameter of $1.5 \mathrm{~cm}$ were mounted in culture chambers (LiveBox2, IVTech, Massarosa, LU, Italy) suitably modified to hold skin explants with the stratum corneum facing up, in contact with air, and the hypodermis facing the lower side, in 
contact with the culture medium (Figure 1) (for a detailed description $\mathrm{see}^{18}$ ).

Two skin samples were treated with $300 \mu \mathrm{L}$ of GEL RHO, and two samples were treated with $300 \mu \mathrm{L}$ of GEL SLN-RHO. Gels were applied on the skin surface from the upper route of administration of the culture chambers (Figure 1). Skin samples were maintained inside the bioreactor under fluid dynamic conditions in the incubator, at $37^{\circ} \mathrm{C}$ in a $5 \% \mathrm{CO}_{2}$ humidified atmosphere, for 2 and $6 \mathrm{~h}$.

Two and $6 \mathrm{~h}$ after administration, skin samples were washed in physiological solution and processed for brightfield and fluorescence microscopy. Control skin samples, collected immediately after excision, were processed in the same way.

\section{Histological analysis}

For histological analysis at brightfield microscopy, skin samples were fixed overnight with $4 \%(\mathrm{v} / \mathrm{v})$ paraformaldehyde in 0.1 $\mathrm{M}$ phosphate buffer at room temperature, dehydrated in ethanol, treated with xylene, and finally embedded in paraffin wax. Seven$\mu \mathrm{m}$-thick sections were de-waxed, rehydrated in graded ethanol and distilled water, and routinely stained with hematoxylin and eosin solution (BioOptica, Milan, Italy) to be observed with an Olympus BX51 microscope equipped with an Olympus Camedia 5050 digital camera (Olympus, Italia Srl, Segrate, MI, Italy).

\section{Confocal images}

For confocal microscopy, skin samples were fixed overnight with $4 \%(\mathrm{v} / \mathrm{v})$ paraformaldehyde in $0.1 \mathrm{M}$ phosphate buffer at room temperature, immersed in a $30 \%(\mathrm{w} / \mathrm{v})$ sucrose solution in PBS, frozen in methyl butane cooled in liquid nitrogen, and finally stored at $-80^{\circ} \mathrm{C}$. Seven- $\mu \mathrm{m}$-thick cryostatic sections were observed with an inverted confocal microscope (TCS SP5 AOBS CMS; Leica GmbH, Wetzlar, Germany). Confocal images were acquired with $543 \mathrm{~nm}$ excitation in the $555-765 \mathrm{~nm}$ wavelength region.

\section{Spectrofluorometric images and analysis}

For the spectrofluorometric analysis, confocal images were performed after excitation at $543 \mathrm{~nm}$ in the $555-765 \mathrm{~nm}$ range, subdividing the range into 21 detection steps width $10 \mathrm{~nm}$ of detection band each. Confocal images were analysed with LASX software (Leica Microsystems). Regions of interests (ROIs) were drawn on the images acquired at $600 \mathrm{~nm}(595-605 \mathrm{~nm})$ from the stratum corneum to the papillary dermis up to a depth of $300 \mu \mathrm{m}$, as well as at the level of the hair bulbs arbitrarily distinguishing between superficial and deep bulbs cutting planes to exemplify a different level of penetration of the formulations through the bulb itself (Figure 2). It is noteworthy that acquisition at $560 \mathrm{~nm}$ (555-665 $\mathrm{nm}$ ) is dominated by laser signal and was used to normalise the fluorescent signal intensity measured in different ROIs and different samples.

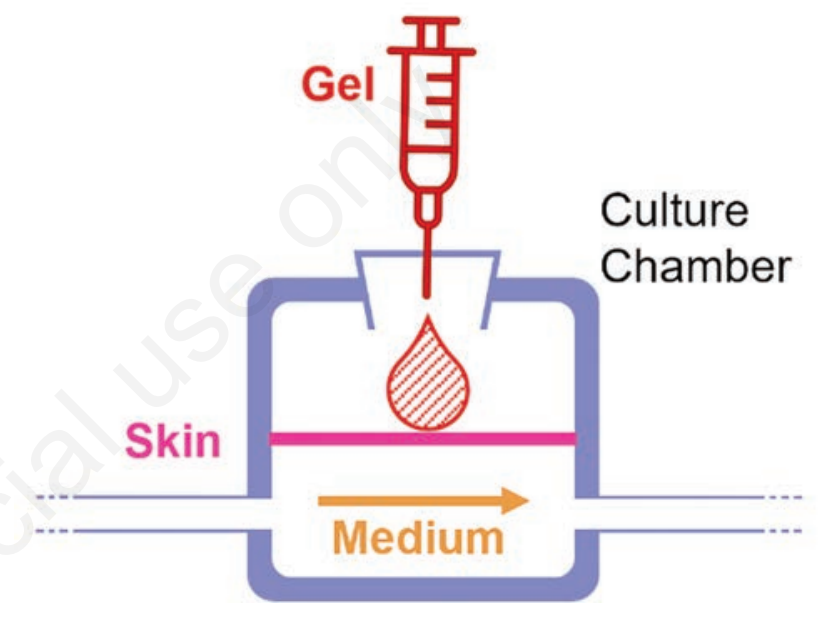

Figure 1. Skin explants were mounted with the stratum corneum facing up in culture chambers to form a lower side, in which culture medium flows, and an upper side, in contact with air and from which the gels were administered.
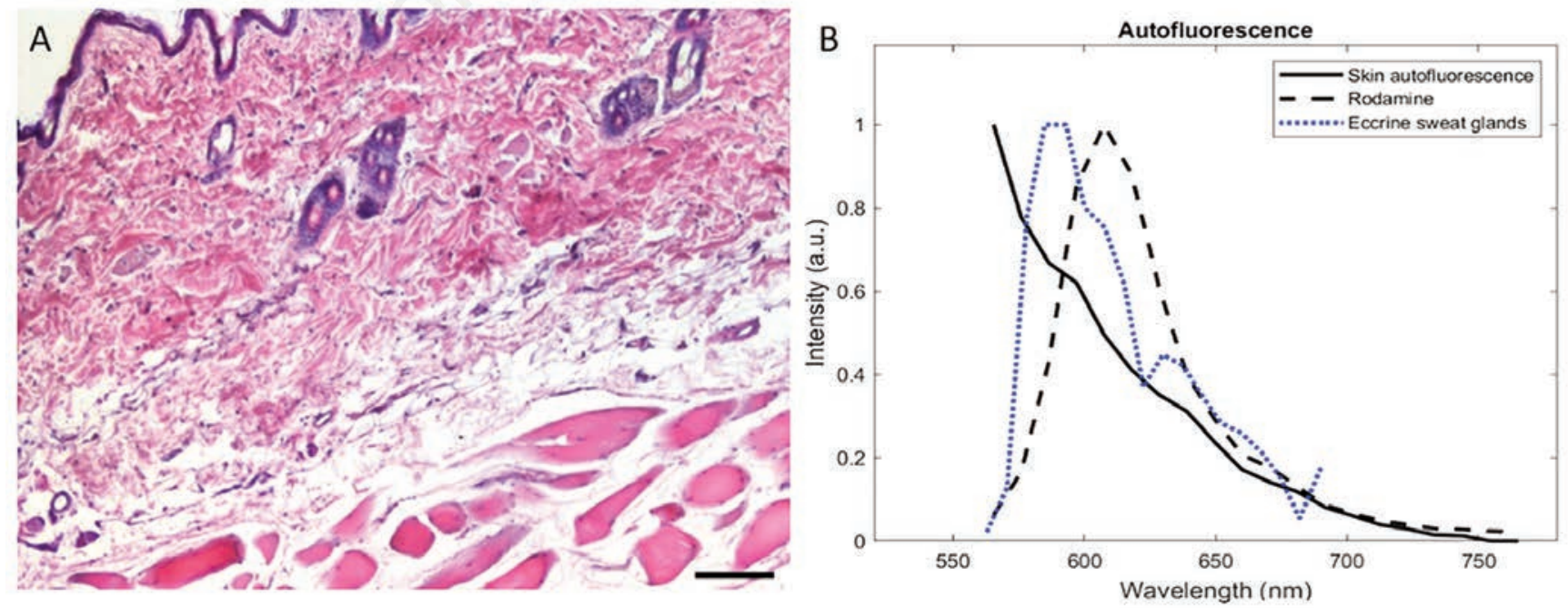

Figure 2. A) Optical micrograph of skin samples incubated for $6 \mathrm{~h}$ inside the bioreactor, stained with H\&E; scale bar: $500 \mu \mathrm{m}$. B) Comparison between spectra of eccrine sweat glands (data obtained from ${ }^{17}$ ), skin autofluorescence and rhodamine normalised at $600 \mathrm{~nm}$. 


\section{Results}

Histological analysis at brightfield microscopy (Figure 2A) demonstrated the good preservation of the skin samples incubated in the bioreactor up to $6 \mathrm{~h}$ after treatment with GEL RHO or GEL SLN-RHO. The two gel formulations did not alter the structural features of the skin; in fact, the layer continuity of epidermis, dermis, and subcutaneous connective tissue, as well as the skin appendages (hair structures, sweat, and sebaceous glands), were all well preserved.

Fluorescence analysis on the samples, performed with a confocal microscope, did not give the expected results, as no significant differences are observed between the control samples and the experimental groups, as regards signal intensity and biodistribution: in all the samples, a signal emission is observed coming from the skin surface, glands and hair bulbs (Figure 3).

In order to assess the skin autofluorescence emission, a spectral analysis was done on control samples as explained in the Materials and Methods section and on rhodamine solution. The spectra are shown in Figure $2 \mathrm{~B}$ together with the eccrine sweat
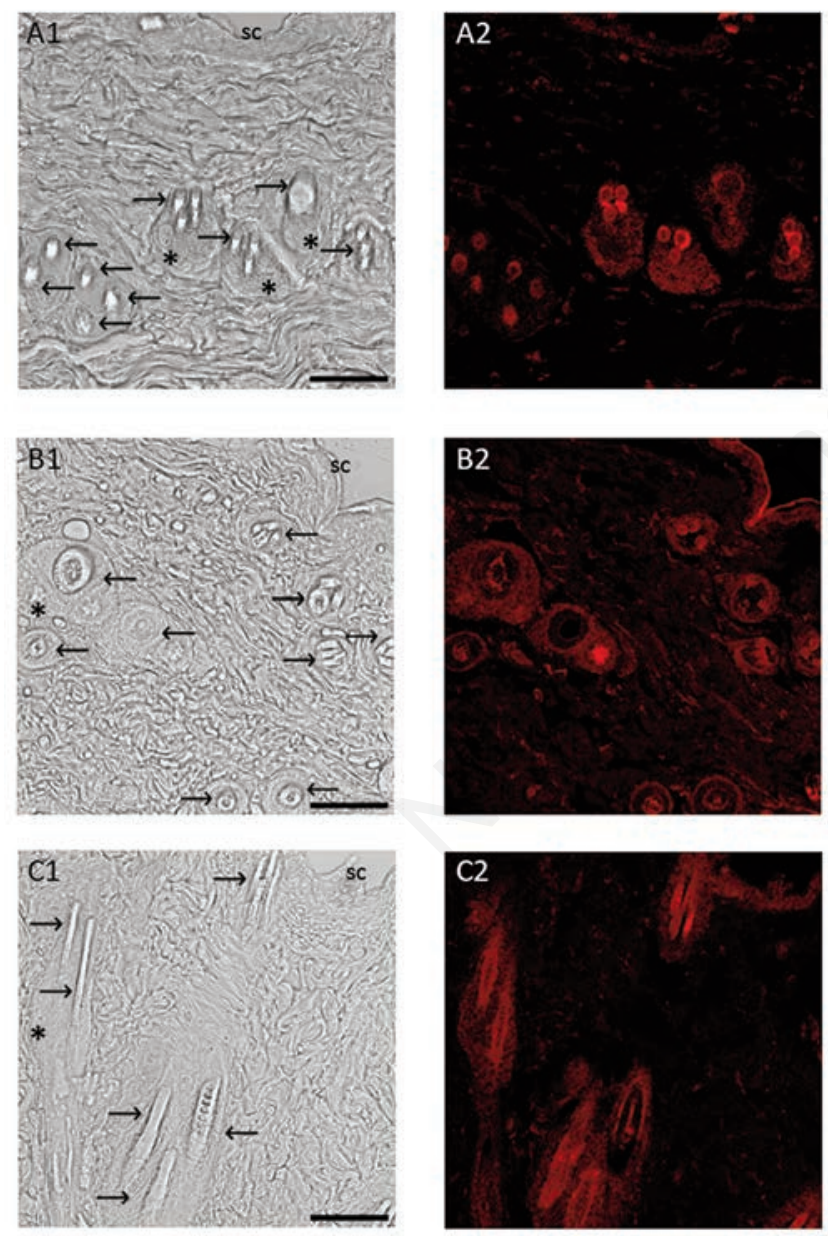

Figure 3. Fluorescent micrographs of control skin samples (A) and after GEL SLN-RHO (B) and GEL RHO (C) administration. Brightfield (left column), and fluorescence images (right column) at $600 \mathrm{~nm}$. It is worth noting the marked autofluorescence in the control skin (A2). No significant differences are observed between the control samples and the experimental groups regarding signal intensity and biodistribution. SC, stratum corneum; arrows, hair bulbs/hair shafts; asterisks, sebaceous glands; scale gland autofluorescence spectrum in the same wavelength region. ${ }^{17}$ In particular, the fluorescent signal of the rhodamine solution was visible and peaked around $600 \mathrm{~nm}$.

Confocal images of control samples revealed a strong autofluorescence signal as visible in Figure 2B, in the range $555-765 \mathrm{~nm}$, i.e., the same range of rhodamine emission.

\section{Epidermis}

At confocal fluorescence microscopy, autofluorescence was observed in the epidermis of control samples under excitation at $543 \mathrm{~nm}$ (Figure 2B) and emission in the range 555-765 nm.

Due to the possible overlap between rhodamine emission and autofluorescence, the acquisition at $560 \mathrm{~nm}$ was included in the spectral acquisition in order to normalise the fluorescent signal with respect to the laser emission. After normalization, the spectrofluorometric measurements showed that fluorescence emission in control samples in the explored wavelength region was negligible compared to the emission observed in treated samples (Figure 4). The spectrofluorometric analysis showed that the fluorescence signal was peaked at $600 \mathrm{~nm}$, being higher in the GEL SLN-RHO samples than in the GEL RHO ones. Moreover, the signal intensity increased in relation to the administration time with both gel formulations.

\section{Papillary dermis}

The emission intensity was measured from the epidermal surface up to $300 \mu \mathrm{m}$. Confocal images showed that, under the keratinized stratum corneum, the fluorescence intensity immediately decreased to the background level with both gel formulations and at both time points (Figure 5).

\section{Glands and bulbs}

We arbitrarily distinguished superficial and deep bulb cutting planes to exemplify two different levels of penetration of the formulations through the bulb itself ("superficial hair bulb" up to 150 $\mu \mathrm{m}$ and "deeper hair bulb" beyond $150 \mu \mathrm{m})$. In the GEL RHO samples, after $2 \mathrm{~h}$, the rhodamine fluorescence was present in the super-

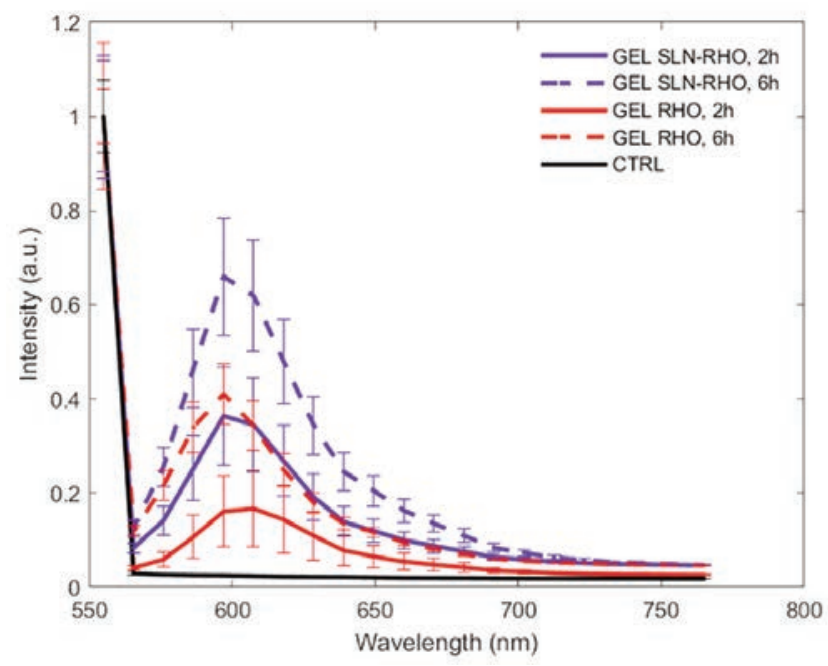

Figure 4. Fluorescence signal in epidermis obtained in 21 wavelength step from 555 to $765 \mathrm{~nm} 10 \mathrm{~nm}$ bandwidth each. GEL SLN-RHO shows a higher signal than GEL RHO. The signal is higher at $6 \mathrm{~h}$ after administration with both gel formulations compared to $\mathbf{2} \mathrm{h}$. Normalization of the fluorescence signal at $\mathbf{5 6 0}$ $\mathrm{nm}$, as explained in the text. Error bars correspond to standard deviation. 
ficial hair bulbs only, while after $6 \mathrm{~h}$, the fluorescence signal was found in the deeper hair bulbs (Figure 6). On the contrary, in the GEL SLN-RHO samples, a stronger signal was detected in the deeper bulbs of the skin already after $2 \mathrm{~h}$, the intensity being similar at $6 \mathrm{~h}$.

In superficial bulbs, fluorescence signal intensity was significantly different between control and administered samples $(p<0.001)$. Moreover, statistically significant differences were found between samples administered with GEL RHO and GEL SLN-RHO after both 2 and $6 \mathrm{~h}(\mathrm{p}<0.005$ and $\mathrm{p}<0.006$, respectively). In the case of GEL RHO formulation, superficial bulbs showed a higher fluorescence signal at $6 \mathrm{~h}$ compared to $2 \mathrm{~h}(\mathrm{p}<0.03)$. Analogously, deep bulbs showed more fluorescent signals in administered samples compared to the control ones $(\mathrm{p}<0.001)$. Furthermore, statistically significant differences were found between samples administered with GEL RHO and GEL SLNRHO after both 2 and $6 \mathrm{~h}(\mathrm{p}<0.001$ in both cases). Finally, deep bulbs administered with GEL SLN-RHO showed a higher fluorescence signal at $6 \mathrm{~h}$ in relation to $2 \mathrm{~h}(\mathrm{p}<0.003)$.

\section{Discussion}

In this work, we investigated the suitability of a new approach for semi-quantitative analysis of fluorescent samples. In particular, we first transcutaneously administered two different gel formula-
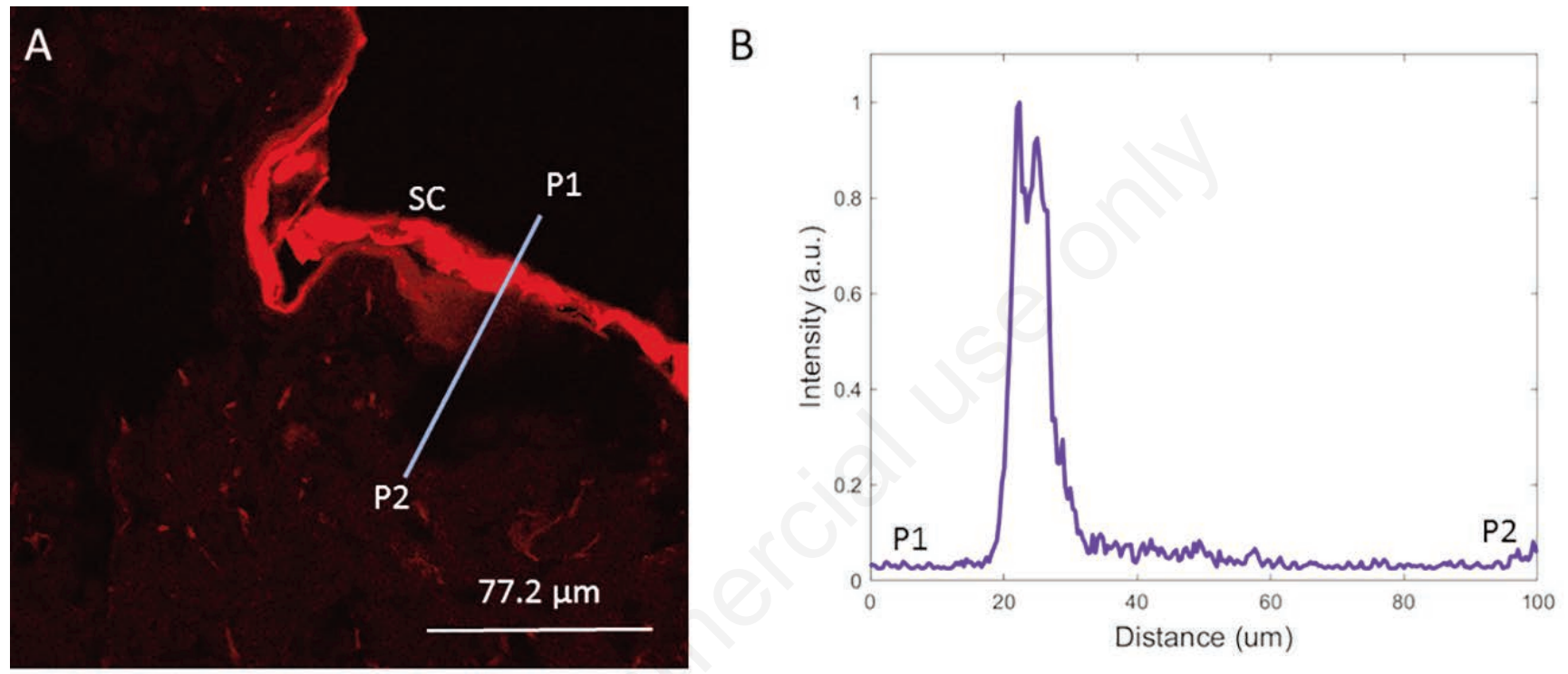

Figure 5. A) Representative image acquired at $600 \mathrm{~nm}$ showing the decrease of fluorescence intensity under the stratum corneum (SC); sample treated with GEL SLN-RHO, $6 \mathrm{~h}$. B) Line profile from P1 and P2 (indicated in A) of fluorescence intensity.
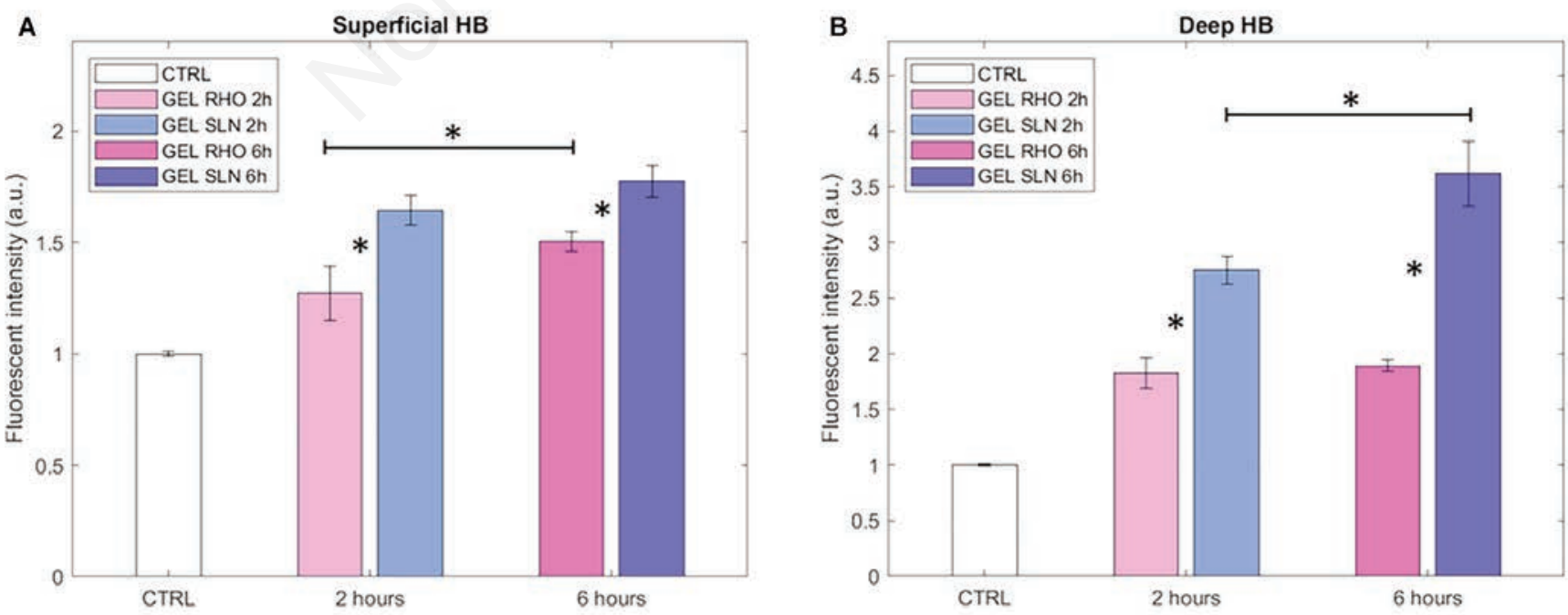

Figure 6. Average signal intensity (normalised at $560 \mathrm{~nm}$ ) at $600 \mathrm{~nm}$ in superficial (A) and deep (B) hair bulbs after 2 and $6 \mathrm{~h}$ after administration. The error bars correspond to the standard error of the mean. 
tions carrying rhodamine to skin explants maintained in a bioreactor. After 2 and $6 \mathrm{~h}$, the samples were analysed with bright field and fluorescence microscopy, and a spectrofluorometric analysis was performed.

Morphological results at bright microscopy confirmed that this bioreactor is a noteworthy device capable of preserving skin explants in vitro in good conditions, maintaining barrier integrity, and constituting a possible technique for the study of the transcutaneous penetration of molecules. ${ }^{18}$

Fluorescence microscopy was used to evaluate signal distribution in skin samples, looking for differences between the two types of formulations in the specific cutaneous regions: epidermis, papillary dermis, and appendages (sweat and sebaceous glands and hair bulbs). Both samples showed a fluorescence signal located in the stratum corneum and hair bulbs, with no apparent difference between the two types of formulations. However, observation at fluorescence microscopy alone cannot provide sufficiently accurate data on the biodistribution of fluorophore in tissues. In fact, an observer cannot subjectively evaluate minor differences in fluorescence intensity, which makes a precise comparison between two or more treatments almost impossible. On the contrary, the spectrofluorometric analysis is an effective semi-quantitative method, which provides the researcher with objective and reliable information and allows detecting even small changes in the fluorescence signals in different tissue layers. Furthermore, the spectral analysis makes it possible to identify specific fluorophores and overcome the autofluorescence issue, which is typically present in some organs, such as the skin. ${ }^{11-13}$

The spectrofluorometric analysis at the epidermis level revealed a higher signal intensity in the upper layer of samples treated with GEL SLN-RHO. However, no passage towards the papillary dermis was seen for both formulations (GEL RHO and GEL SLN-RHO). The above indicates that there is no penetration through the epidermis. Conversely, both formulations proved to pass through the trans-appendages pathway and, again, higher signal intensity was found for GEL SLN-RHO, even at the deeper level of the bulb, indicating that already after $2 \mathrm{~h}$, this formulation can reach the deep layers of the skin dermis better than the GEL RHO.

In sum, the GEL SLN-RHO formulation gave better results than GEL RHO. We can advance two hypotheses to explain this finding: i) SLNs are able to protect the fluorophore from enzymatic and chemical degradation and/or quenching, as widely reported in the literature, ${ }^{8,22}$; ii) SLNs are a more effective and faster carrier for transporting molecules through skin layers than a pure gel. From a technical point of view, our spectrofluorimetric analysis proved to be a reliable method to identify a fluorophore and study its biodistribution into an organ characterised by a high autofluorescence by using its own emission spectrum to distinguish the fluorophore signal from the background. Moreover, this method is also suitable for any type of fluorophore; in fact, the autofluorescence spectrum covers a broad wavelength overlapping the rhodamine signaland other fluorophores. Furthermore, this method was particularly useful because it allowed us to distinguish transcutaneous penetration capacities of the two formulations thanks to normalization with a constant signal, represented by the laser's intensity at a fixed wavelength. Generally, the parameters of the laser and photomultipliers are modified to optimise the observation and the image, providing a reliable method for comparing different samples.

In conclusion, the association between histological techniques and spectrofluorometry may be particularly suitable in nanomedicine for studying the distribution of diagnostic or therapeutic agents. By this approach, we were able to identify different biodistributions between the used formulations and demonstrate that SLNs thickened with polymer, resulting in a nanoparticulate gel, are suitable drug carriers for transcutaneous administration.

\section{Acknowledgments}

This work was supported by the University of Verona (Joint Projects 2017). EC was a Ph.D. student receiving a fellowship from the Doctoral Program "Nanoscience and Advanced Technologies" of the University of Verona. Confocal microscopy and spectrofluorimetry were performed at the Centro Piattaforme Tecnologiche of the University of Verona.

\section{References}

1. Berti JJ, Lipsky JJ. Transcutaneous drug delivery: a practical review. Mayo Clin Proc 1995;70:581-6.

2. dos Anjos JL, de Sousa Neto D, Alonso A. Effects of ethanol/1menthol on the dynamics and partitioning of spin-labeled lipids in the stratum corneum. Eur J Pharm Biopharm 2007;67:406-12.

3. Kristl J, Teskac K, Grabnar PA. Current view on nanosized solid lipid carriers for drug delivery to the skin. J Biomed Nanotechnol 2010;6:529-42.

4. Abdel-Mottaleb MM, Try C, Pellequer Y, Lamprecht A. Nanomedicine strategies for targeting skin inflammation. Nanomedicine (Lond) 2014;9:1727-43.

5. Kim BY, Rutka JT, Chan WC. Nanomedicine. N Engl J Med 2010;16;363:2434-43.

6. Baetke SC, Lammers T, Kiessling F. Applications of nanoparticles for diagnosis and therapy of cancer $\mathrm{Br} \mathrm{J}$ Radiol 2015;88:20150207.

7. Sguizzato M, Mariani P, Ferrara F, Drechsler M, Hallan SS, Huang N, et al. Nanoparticulate gels for cutaneous administration of caffeic acid. Nanomaterials (Basel) 2020;10:961.

8. Wolfbeis OS. An overview of nanoparticles commonly used in fluorescent bioimaging. Chem Soc Rev 2015;21;44:4743-68.

9. Ruedas-Rama MJ, Walters JD, Orte A, Hall EA. Fluorescent nanoparticles for intracellular sensing: a review. Anal Chim Acta 2012;751:1-23.

10. Zhang LW, Monteiro-Riviere NA. Use of confocal microscopy for nanoparticle drug delivery through skin. J Biomed Opt 2013;18:061214.

11. Roberts MS, Dancik Y, Prow TW, Thorling CA, Lin LL, Grice JE, et al. Non-invasive imaging of skin physiology and percutaneous penetration using fluorescence spectral and lifetime imaging with multiphoton and confocal microscopy. Eur J Pharm Biopharm 2011;77:469-88.

12. Wu Y, Qu JY. Autofluorescence spectroscopy of epithelial tissues. J Biomed Opt 2006;11:054023.

13. Giovannacci I, Magnoni C, Vescovi P, Painelli A, Tarentini E, Meleti M. Which are the main fluorophores in skin and oral mucosa? A review with emphasis on clinical applications of tissue autofluorescence. Arch Oral Biol 2019;105:89-98.

14. Andersson-Engels S, Klinteberg C, Svanberg K, Svanberg S. In vivo fluorescence imaging for tissue diagnostics. Phys Med Biol 1997;42:815-24.

15. Bouchard MB, MacLaurin SA, Dwyer PJ, Mansfield J, Levenson R, Krucker T. Technical considerations in longitudinal multispectral small animal molecular imaging. J Biomed Opt 2007;12:051601.

16. Croce AC, Bottiroli G. Autofluorescence spectroscopy and imaging: a tool for biomedical research and diagnosis. Eur J 
Histochem 2014;58:2461.

17. Zhao HL, Chen Y, Zhao HJ, Tan ZJ, Zhang CP, Fu XB, et al. Autofluorescence of eccrine sweat glands. Skin Res Technol 2016;22:98-103.

18. Cappellozza E, Zanzoni S, Malatesta M, Calderan L. Integrated microscopy and metabolomics to test an innovative fluid dynamic system for skin explants in vitro. Microsc Microanal 2021;27:923-34.

19. Mathes SH, Ruffner H, Graf-Hausner U. The use of skin models in drug development. Adv Drug Deliv Rev 2014;69-70:81102.
20. Abaci HE, Guo Z, Doucet Y, Jacków J, Christiano A. Next generation human skin constructs as advanced tools for drug development. Exp Biol Med (Maywood) 2017;242:1657-68.

21. Sguizzato M, Valacchi G, Pecorelli A, Boldrini P, Simelière F, Huang N, et al. Gallic acid loaded poloxamer gel as new adjuvant strategy for melanoma: A preliminary study. Colloids Surf B Biointerfaces 2020;185:110613.

22. Reisch A, Klymchenko AS. Fluorescent polymer nanoparticles based on dyes: Seeking brighter tools for bioimaging. Small 2016;12:1968-92.

Received for publication: 2 September 2021. Accepted for publication: 17 JAnuary 2022.

This work is licensed under a Creative Commons Attribution-NonCommercial 4.0 International License (CC BY-NC 4.0).

(C) Copyright: the Author(s), 2022

Licensee PAGEPress, Italy

European Journal of Histochemistry 2022; 66:3321

doi:10.4081/ejh.2022.3321 